\title{
The empirical equilibrium structure of diacetylene ${ }^{\star}$
}

\author{
Sven Thorwirth ${ }^{\text {a }}$ Michael E. Harding ${ }^{b}$ Dirk Muders ${ }^{a}$ \\ Jürgen Gauss ${ }^{b}$ \\ a Max-Planck-Institut für Radioastronomie, Auf dem Hügel 69, 53121 Bonn, \\ Germany \\ ${ }^{\mathrm{b}}$ Institut für Physikalische Chemie, Universität Mainz, 55099 Mainz, Germany
}

\begin{abstract}
High-level quantum-chemical calculations are reported at the MP2 and CCSD(T) levels of theory for the equilibrium structure and the harmonic and anharmonic force fields of diacetylene, $\mathrm{H}-\mathrm{C} \equiv \mathrm{C}-\mathrm{C} \equiv \mathrm{C}-\mathrm{H}$. The calculations were performed employing Dunning's hierarchy of correlation-consistent basis sets cc-pVXZ, ccpCVXZ, and cc-pwCVXZ, as well as the ANO2 basis set of Almlöf and Taylor. An empirical equilibrium structure based on experimental rotational constants for thirteen isotopic species of diacetylene and computed zero-point vibrational corrections is determined $\left(r_{e}^{e m p}: r_{\mathrm{C}-\mathrm{H}}=1.0615 \AA, r_{\mathrm{C} \equiv \mathrm{C}}=1.2085 \AA, r_{\mathrm{C}-\mathrm{C}}=1.3727 \AA\right)$ and in good agreement with the best theoretical structure $(\operatorname{CCSD}(\mathrm{T}) / \mathrm{cc}-\mathrm{pCV} 5 \mathrm{Z}$ : $\left.r_{\mathrm{C}-\mathrm{H}}=1.0617 \AA, r_{\mathrm{C} \equiv \mathrm{C}}=1.2083 \AA, r_{\mathrm{C}-\mathrm{C}}=1.3737 \AA\right)$. In addition, the computed fundamental vibrational frequencies are compared with the available experimental data and found in satisfactory agreement.
\end{abstract}

Key words: Diacetylene, Butadiyne, Rotation-vibration interaction, Anharmonic force field, Equilibrium structure

\section{Introduction}

Diacetylene (DiA), $\mathrm{H}-\mathrm{C} \equiv \mathrm{C}-\mathrm{C} \equiv \mathrm{C}-\mathrm{H}$, the simplest polyacetylene, is of importance in organic chemical synthesis [1] and is also an abundant astronomical

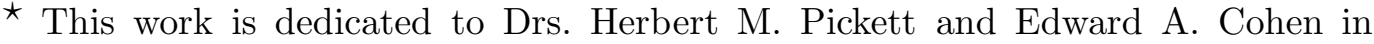
recognition of their many valuable contributions to the field of laboratory molecular spectroscopy.

Email address: sthorwirth@mpifr-bonn.mpg.de (Sven Thorwirth).
} 
species. In space, it was detected through infrared spectroscopy in the atmosphere of Saturn's moon Titan [2] and in the protoplanetary nebula CRL618 [3]. In the laboratory, it has been the subject of numerous spectroscopic studies in the infrared and also in the microwave regime (see Refs. [4,5] and references therein). Spectroscopic data were also used to evaluate its structural parameters using different approaches. An advanced experimental near-equilibrium $\left(r_{m}^{\rho}\right)_{c o r r}$ structure was reported by Tay et al. [4] making use of experimental rotation-vibration data of a total of nine different isotopic species. Additionally, various quantum-chemical calculations were performed with an empirically corrected frozen-core CCSD(T)/cc-pVQZ calculation [6] representing the highest theoretical level employed to date.

The equilibrium structure of DiA is of interest, as it represents one of the simplest systems with conjugated carbon-carbon multiple bonds. The question is how conjugation affects the structure and in particular shortens the central carbon-carbon single bond. It is well known that low-level quantum-chemical calculations are not reliable in this respect, with Hartree-Fock calculations typically underestimating the conjugation and density-functional theory overestimating it. High-level calculations, preferably at the coupled-cluster (CC) level [7], are needed for reliable predictions. To determine the accuracy of the $\mathrm{CC}$ calculations, it is essential to provide an equilibrium structure based on experimental data.

In a recent Fourier transform microwave spectroscopic study, the rotational spectra of four new isotopologs of monodeutero diacetylene were characterized [5] raising the number of known isotopic species of DiA to a total of thirteen. In the present work, we report an empirical (or semi-experimental) equilibrium structure for DiA together with corresponding high-level CC results. The key to the empirical structure is the calculation of accurate anharmonic force fields which enables the determination of individual zero-point vibrational contributions

$$
\Delta B_{0}^{\text {calc }}=\sum_{i} \alpha_{i}\left(d_{i} / 2\right)
$$

for each isotopolog. In Eq. (1), the vibrational correction $\Delta B_{0}^{\text {calc }}$ is given as the sum of calculated rotation-vibration interaction constants $\alpha_{i}$ with $d_{i}$ as the degeneracy being 1 for non-degenerate (stretching) vibrations and 2 for doubly degenerate (bending) vibrations. With those corrections, it is possible to obtain empirical equilibrium values for the rotational constants according to

$$
B_{e}^{e m p}=B_{0}^{e x p}+\Delta B_{0}^{c a l c} .
$$

The equilibrium structure is then obtained through a least-squares fit of the 
structural parameters to the estimated equilibrium moments of inertia $I_{e}$ (see, e.g., Ref. [8]). In case of DiA these are the three bond distances $r_{\mathrm{C}-\mathrm{H}}, r_{\mathrm{C} \equiv \mathrm{C}}$, and $r_{\mathrm{C}-\mathrm{C}}$.

\section{Computational Details}

Quantum-chemical calculations were performed using second-order MøllerPlesset (MP2) perturbation theory as well as the coupled-cluster singles and doubles (CCSD) approach augmented by a perturbative treatment of triple excitations (CCSD $(\mathrm{T})$ ) [9]. The calculations were carried out using basis sets from Dunning's hierarchy of correlation-consistent bases [10]. To be more specific, the cc-pVXZ $(X=\mathrm{D}-6)$ sets [10] have been used in frozen-core (fc) MP2 and $\operatorname{CCSD}(\mathrm{T})$ calculations, while the cc-pCVXZ $(X=\mathrm{T}-5)$ and cc-pwCV $X Z$ $(X=\mathrm{T}, \mathrm{Q})$ sets $[11,12]$ have been chosen for the all-electron calculations. Additional calculations were also performed with atomic natural orbital (ANO) basis sets from Almlöf and Taylor [13]. The latter basis set, ANO2 1 , was employed within the fc approximation.

Equilibrium geometries were optimized using analytic gradient techniques [14]. Harmonic, cubic, and semidiagonal quartic force fields were then obtained using analytic second-derivative techniques $[8,15]$. The required third and fourth derivatives for the anharmonic force fields were determined by additional numerical differentiation as described in Refs. $[8,16]$. The vibration-rotation interaction constants $\alpha_{i}$ were calculated using formulas given in Ref. [17] based on lowest-order rovibrational perturbation theory. Recalculation of the cubic force field is avoided by transforming the computed force field for the main isotopolog to the corresponding normal coordinate representations of the other isotopologs. Since this is not possible for the semidiagonal quartic force field, fundamental frequencies, again determined using lowest-order rovibrational perturbation theory [17], are only reported for the main isotopic species.

All calculations have been performed with the Mainz-Austin-Budapest version of ACESII [18]; some of the expensive (larger) calculations were made possible by using our recent parallel implementation of CC energy as well as first and second derivative calculations [19].

1 The ANO2 set consists of a $(13 \mathrm{~s} 8 \mathrm{p} 6 \mathrm{~d} 4 \mathrm{f} 2 \mathrm{~g} / 5 \mathrm{~s} 4 \mathrm{p} 3 \mathrm{~d} 2 \mathrm{f} 1 \mathrm{fg})$ contraction for $\mathrm{C}$ and a (8s6p4d3f/4s3p2d1f) contraction for H. 


\section{Source of Experimental Data and Least-Squares Fit}

Experimental $B_{0}$ values of thirteen isotopologues studied through rotationvibration [4,20] and rotational spectroscopy [5] are collected in Table 1 . The least-squares fits for the structural parameters have been performed including the data for all thirteen isotopologues. The fits have been performed with respect to the moments of inertia using the same weight for all considered isotopic species.

\section{Results and Discussion}

Table 1 summarizes in addition to the experimental rotational constants $B_{0}$ also the corresponding calculated vibrational corrections as obtained at the (fc)MP2/cc-pVTZ, (fc)CCSD(T)/cc-pVTZ, (fc)CCSD(T)/cc-pVQZ, CCSD(T)/ccpCVQZ, and (fc) CCSD(T)/ANO2 levels of theory. The vibrational corrections $\Delta B_{0}$ have been also computed with other basis sets (not given here explicitly). Analysis of all these calculations allows one to draw the following conclusions (numbers given in the following with respect to the parent isotopic species): (a) the use of polarized split-valence basis sets (i.e., cc-pVXZ) in all-electron calculations leads to too large corrections (e.g. $3.80 \mathrm{MHz}$ for MP2/cc-pVTZ in comparison to $2.1 \mathrm{MHz}$ for (fc)MP2/cc-pVTZ); (b) MP2 calculations overestimate the vibrational corrections in comparison with $\operatorname{CCSD}(\mathrm{T})$ computations (e.g., $2.1 \mathrm{MHz}$ for (fc)MP2/cc-pVTZ and $0.91 \mathrm{MHz}$ for (fc)CCSD(T)/ccpVTZ); (c) the use of quadruple-zeta basis sets is recommended, as the smaller cc-pVTZ and cc-pCVTZ basis sets yield vibrational corrections with rather large errors (e.g. $0.91 \mathrm{MHz}$ for (fc)CCSD(T)/cc-pVTZ in comparison with 1.92 $\mathrm{MHz}$ for (fc)CCSD(T)/cc-pVQZ and $2.22 \mathrm{MHz}$ for (fc)CCSD(T)/cc-pCVTZ in comparison with $1.84 \mathrm{MHz}$ for (fc) CCSD(T)/cc-pCVQZ); (d) the use of the ANO2 set yields similar results as the corresponding cc-pVQZ calculations.

From this analysis we also conclude that our computed vibrational corrections (at the CCSD(T)/cc-pCVQZ and (fc)CCSD(T)/cc-pVQZ levels) should have an accuracy of about 0.2 to $0.3 \mathrm{MHz}$. This accuracy is sufficient for obtaining an accurate equilibrium structure of DiA from the empirical $B_{e}$ rotational constants and, in turn, it is more than adequate for a theoretical prediction of ground state rotational constants $B_{0}$. For the latter, the errors in the theoretical determination of high-level $B_{e}$ values due to remaining basis-set errors and still missing electron-correlation contributions certainly are larger than the zero-point vibrational correction $\Delta B_{0}^{\text {calc }}[21]$.

Table 2 gathers the new extensive set of structural parameters for DiA. There, we report the different empirical structures of DiA as obtained from using the 
vibrational corrections given in Table 1 and the pure theoretical structures based on high-level CC calculations using a variety of basis sets. In addition, structural parameters from the literature are given $[4,5,6]$.

Comparing our five different empirical $r_{e}^{e m p}$ structures, we note that values of $1.062 \AA, 1.208 \AA$, and $1.373 \AA$ are obtained for the $\mathrm{C}-\mathrm{H}$ distance, the $\mathrm{C} \equiv \mathrm{C}$ triple bond, and $\mathrm{C}-\mathrm{C}$ single bond, respectively, irrespective of the used vibrational corrections. The differences in the vibrational corrections affects the bond lengths only in the fourth decimal. Nevertheless, the changes when going from the cc-pVTZ to the cc-pVQZ or cc-pCVQZ corrections are not entirely negligible and we consider the results obtained with the $(\mathrm{fc}) \operatorname{CCSD}(\mathrm{T}) / \mathrm{cc}-$ pVQZ, CCSD (T)/cc-pCVQZ, and CCSD(T)/ANO2 corrections the most reliable. The high quality of these three sets of equilibrium distances is also seen through the fact that the residuals in the fits are as small as $6 \mathrm{kHz}$ for the maximum and $0.7 \mathrm{kHz}$ for the root mean-square deviations. The statistical uncertainties in all of the fits are small being $0.0004 \AA$ (CC single bond), $0.0003 \AA$ (CC triple bond), and $0.0001 \AA$ ( $\mathrm{CH}$ bond) and hence in all cases below $0.001 \AA$.

Comparison of the derived empirical $r_{e}$ structures with the pure theoretical geometries reveals a good agreement with the results obtained at our best quantum-chemical level (CCSD(T)/cc-pCV5Z). With $0.001 \AA$ and less the discrepancies are in the expected range and probably due to the neglect of higher excitations in the $\mathrm{CC}$ treatment [22]. A closer analysis of the extensive set of theoretical data in Table 2 furthermore demonstrates the importance of core correlation, as the (fc) $\operatorname{CCSD}(\mathrm{T})$ calculations yield too long bond distances (by about $0.0015 \AA$ for the $\mathrm{CH}$ distances and $0.003 \AA$ for the CC distances). However, core-correlation effects can be accurately treated using an additivity assumption, since the distances obtained via

$$
r_{e} \simeq r_{e}(\mathrm{pV} 6 \mathrm{Z}, \mathrm{fc})+r_{e}(\mathrm{pwCVQZ}, \mathrm{ae})-r_{e}(\mathrm{pwCVQZ}, \mathrm{fc})
$$

are $r_{\mathrm{C}-\mathrm{H}}=1.0618 \AA, r_{\mathrm{C} \equiv \mathrm{C}}=1.2083 \AA$, and $r_{\mathrm{C}-\mathrm{C}}=1.3737 \AA$ and hence within $10^{-4} \AA$ of the CCSD(T)/cc-pCV5Z results.

For the sake of completeness, Table 2 also gives parameters from other equilibrium or near-equilibrium structure determinations. Reasonable agreement is found between the best empirical equilibrium structures $r_{e}^{e m p}((\mathrm{fc}) \operatorname{CCSD}(\mathrm{T}) / \mathrm{cc}-$ pVQZ, $\operatorname{CCSD}(\mathrm{T}) / \mathrm{cc}-\mathrm{pCVQZ}$, and (fc) $\operatorname{CCSD}(\mathrm{T}) / \mathrm{ANO} 2)$ and the near-equilibrium $\left(r_{m}^{\rho}\right)_{c o r r}$ structure of Tay et al. [4] with the biggest discrepancy being in the central $\mathrm{C}-\mathrm{C}$ bond. Somewhat larger discrepancies are found in comparison against the $r_{e}$ structure from Ref. [5]. However, the latter structure was only based on a rough empirical correction for the difference between the $r_{s}$ and $r_{e}$ structure of $\mathrm{HC}_{3} \mathrm{~N}$ (without determining the vibrational corrections explicitly) and hence is not comparable from a rigorous point of view. Good agreement 
is found for the structure given by Botschwina and Puzzarini [6] where a theoretical structure obtained at the $\operatorname{CCSD}(\mathrm{T}) / \mathrm{cc}-\mathrm{pVQZ}$ level of theory was empirically corrected through comparison against an empirical equilibrium structure of $\mathrm{HC}_{3} \mathrm{~N}$. Finally, a comparison of the $r_{e}^{e m p}$ structures against the $r_{0}$ structure in Table 2 reveals the significance of including vibrational corrections in structural determinations. In the latter structure the $\mathrm{CH}$ distances are significantly shorter (by about $0.005 \AA$ ) whereas the CC single bond is longer (by about $0.002 \AA$ ) than in the $r_{e}^{e m p}$ structures. A similar conclusion also holds for the comparison with the $r_{s}$ structure reported in Ref. [5] for HCCCCD.

Comparison of the $r_{e}^{e m p}$ parameters of diacetylene with those of similar molecules reveals that the $\mathrm{C}-\mathrm{H}$ bond lengths found in diacetylene $(1.6016 \AA$, this work), fluoroacetylene (1.6014 $\AA$, Ref. [6]), methyldiacetylene (1.6013 $\AA$, Ref. [23]), acetylene (1.0618 $\AA$, Ref. [24]), $\mathrm{HC}_{3} \mathrm{~N}$ (1.0623 $\AA$, Ref. [25]) and methylacetylene (1.061 $\AA$, Ref. [26]) are practically identical. Clearly, conjugation effects in DiA manifest themselves in the $\mathrm{C} \equiv \mathrm{C}$ bond length: whereas in acetylene a value of $1.2029(1) \AA$ is found [24], the $\mathrm{C} \equiv \mathrm{C}$ triple bond is significantly longer in diacetylene, namely $1.2084 \AA$. The latter value is in good agreement with those found in similar conjugated systems. Recent examples are $\mathrm{CC}$ distances in the ethynyl group of the substituted diacetylenes $\mathrm{HC}_{4} \mathrm{~F}$ and $\mathrm{CH}_{3} \mathrm{C}_{4} \mathrm{H}(1.2080 \AA[6]$ and $1.2085 \AA$ [23], respectively) and also the $\mathrm{C} \equiv \mathrm{C}$ distances in branched species such as cis-Hex-3-ene-1,5-diyne and (Z)-pent-2en-4-ynenitrile (1.208(3) $\AA$ [27] and 1.207(3) $\AA$ [28]). Given the prototypical $\mathrm{C}=\mathrm{C}$ equilibrium bond length in ethylene of $1.3305 \AA$ [29]) the formal $\mathrm{C}-\mathrm{C}$ single bond length in DiA of $1.3727 \AA$ found here is very short (and comparable to monofluorodiacetylene $(1.3729 \AA)$ and methyldiacetylene (1.3734 $\AA$ ) indicative of some double-bond character. Furthermore, conjugation i.e. $\pi$ electron delocalization is more pronounced in $\mathrm{DiA}$ (and $\mathrm{HC}_{4} \mathrm{~F} / \mathrm{CH}_{3} \mathrm{C}_{4} \mathrm{H}$ ) than in $\mathrm{HC}_{3} \mathrm{~N}$ where the $\mathrm{C} \equiv \mathrm{C}$ bond is found to be shorter (intermediate between acetylene and $\mathrm{DiA}$ ) and the $\mathrm{C}-\mathrm{C}$ bond longer compared to DiA (Table 1).

Several infrared studies of the vibrational fundamentals, overtones and hot bands of DiA are found in the literature (see Ref. [4] and references therein). In Table 3 we report harmonic and fundamental frequencies of DiA as obtained through $\operatorname{CCSD}(\mathrm{T})$ calculations of the cubic and semi-diagonal quartic force fields using the cc-pVTZ, cc-pVQZ, and ANO2 basis sets in the fc approximation and the cc-pCVQZ basis correlating all-electrons. The accuracy of the reported fundamental frequencies obtained using Dunning's quadruple-zeta basis sets is probably only a couple of wavenumbers, as it turned out impossible to converge the required CC second-derivative calculations at the displaced points with very tight convergence thresholds. In addition, we also report the corresponding (fc)MP2/cc-pVTZ results. Comparison of the theoretical anharmonic (fundamental) frequencies against the experimental values collected in Ref. [30] reveals that the results obtained with the cc-pVXZ $(X=\mathrm{T}, \mathrm{Q})$ sets 
turn out less reliable, while better agreement with experiment is seen for the other two basis sets. For both, the cc-pCVQZ and the ANO2 set, the computed frequencies match the experimental values within about $10 \mathrm{~cm}^{-1}$. This finding again documents the suitability of the ANO basis sets for frequency calculations, while the Dunning basis sets (at least when using the valence sets) show some deficiencies [31,32,33,34,35].

\section{Conclusions}

The equilibrium structure of diacetylene has been determined based on the combination of experimental rotational constants $B_{0}$ of thirteen isotopic species and zero-point vibrational corrections $\Delta B_{0}$ calculated at various quantumchemical levels. The empirical equilibrium structures obtained agree to within $10^{-3} \AA$ irrespective of the theoretical level employed. From the present study, the new recommended equilibrium structure of DiA is $r_{\mathrm{C}-\mathrm{H}}=1.0615 \AA$, $r_{\mathrm{C} \equiv \mathrm{C}}=1.2085 \AA$, and $r_{\mathrm{C}-\mathrm{C}}=1.3727 \AA$. This structure is in good agreement with complementary high-level CC calculations performed here. The structural parameters at the highest level of theory (CCSD $(\mathrm{T}) / \mathrm{cc}-\mathrm{pCV} 5 \mathrm{Z})$ are $r_{\mathrm{C}-\mathrm{H}}=1.0617 \AA, r_{\mathrm{C} \equiv \mathrm{C}}=1.2083 \AA, r_{\mathrm{C}-\mathrm{C}}=1.3737 \AA$.

Evaluation of cubic and semi-diagonal quartic force fields calculated at the CCSD(T)/cc-pCVQZ and (fc)CCSD(T)/ANO2 levels yielded harmonic and anharmonic vibrational frequencies being in good agreement with experiment, while corresponding (fc)MP2 and (fc) CCSD(T) calculations with the cc-pVXZ $(X=\mathrm{T}, \mathrm{Q})$ sets are less satisfactory.

\section{Acknowledgment}

This work at Mainz has been supported by the Deutsche Forschungsgemeinschaft (DFG) and the Fonds der Chemischen Industrie.

\section{References}

[1] I. A. Maretina, B. A. Trofimov, Russ. Chem. Rev. 69 (2000) 591-608.

[2] V. G. Kunde, A. C. Aikin, R. A. Hanel, D. E. Jennings, W. C. Maguire, R. E. Samuelson, Nature 292 (1981) 686-688.

[3] J. Cernicharo, A. M. Heras, A. G. G. M. Tielens, J. R. Pardo, F. Herpin, M. Guélin, L. B. F. M. Waters, Astrophys. J. 546 (2001) L123-L126.

[4] G. Tay, G. F. Metha, F. Shanks, D. McNaughton, Struct. Chem. 6 (1995) 47-55.

[5] K. Matsumura, R. D. Suenram, F. J. Lovas, T. Tanaka, J. Mol. Spectrosc. 240 (2006) 120-126. 
[6] P. Botschwina, C. Puzzarini, J. Mol. Spectrosc. 208 (2001) 292-294.

[7] J. Gauss, Coupled Cluster Theory, in: P. v. R. Schleyer, N. L. Allinger, T. Clark, J. Gasteiger, P. A. Kollmann, H. F. Schaefer, P. R. Schreiner (Eds.), Encyclopedia of Computational Chemistry, Wiley, Chichester, 1998, pp. 615636.

[8] J. F. Stanton, J. Gauss, Int. Rev. Phys. Chem. 19 (2000) 61-95.

[9] K. Raghavachari, G. W. Trucks, J. A. Pople, M. Head-Gordon, Chem. Phys. Lett. 157 (1989) 479-483.

[10] T. H. Dunning, J. Chem. Phys. 90 (1989) 1007-1023.

[11] D. E. Woon, T. H. Dunning, J. Chem. Phys. (1995) 4572-4585.

[12] K. A. Peterson, T. H. Dunning, J. Chem. Phys. 117 (2002) 10548-10560.

[13] J. Almlöf, P. R. Taylor, J. Chem. Phys. 86 (1987) 4070-4077.

[14] J. D. Watts, J. Gauss, R. J. Bartlett, Chem. Phys. Lett. 1-2 (1992) 1-7.

[15] J. Gauss, J. F. Stanton, Chem. Phys. Lett. 276 (1997) 70-77.

[16] J. F. Stanton, C. L. Lopreore, J. Gauss, J. Chem. Phys. 108 (1998) 7190-7196.

[17] I. M. Mills, Vibration-Rotation Structure in Asymmetric- and Symmetric-Top Molecules, in: K. N. Rao, C. W. Mathews (Eds.), Molecular Spectroscopy: Modern Research, Academic Press, New York, 1972, pp. 115-140.

[18] J. F. Stanton, J. Gauss, J. D. Watts, P. G. Szalay, R. J. Bartlett, with contributions from A. A. Auer, D. B. Bernholdt, O. Christiansen, M. E. Harding, M. Heckert, O. Heun, C. Huber, D. Jonsson, J. Jusélius, W. J. Lauderdale, T. Metzroth, C. Michauk, D. R. Price, K. Ruud, F. Schiffmann, A. Tajti, M. E. Varner, J. Vázquez and the integral packages: MOLECULE (J. Almlöf and P. R. Taylor), PROPS (P. R. Taylor), and ABACUS (T. Helgaker, H. J. Aa. Jensen, P. Jørgensen, and J. Olsen). See, also J. F. Stanton, J. Gauss, J. D. Watts, W. J. Lauderdale, R. J. Bartlett, Int. J. Quantum Chem. Symp. 26 (1992), 879. Current version see http://www.aces2.de.

[19] M. E. Harding, T. Metzroth, J. Gauss, A. A. Auer, J. Chem. Theory Comput. 4 (2008) 64-74.

[20] E. Arié, J. W. C. Johns, J. Mol. Spectrosc. 155 (1992) 195-204.

[21] C. Puzzarini., M. Heckert, J. Gauss, submitted to J. Chem. Phys.

[22] M. Heckert, M. Kállay, J. Gauss, Mol. Phys. 103 (2005) 2109-2115.

[23] G. Cazzoli, L. Cludi, M. Contento, C. Puzzarini, submitted to J. Mol. Spectrosc.

[24] G. Cazzoli, C. Puzzarini, L. Fusina, F. Tamassia, J. Mol. Spectrosc. 247 (2008) $115-118$.

[25] P. Botschwina, Mol. Phys. 103 (2005) 1441-1460. 
[26] M. Le Guennec, J. Demaison, G. Wlodarczak, C. J. Marsden, J. Mol. Spectrosc. 160 (1993) 471-490.

[27] R. J. McMahon, R. J. Halter, R. J. Fimmen, R. J. Wilson, S. A. Peebles, R. L. Kuczkowski, J. F. Stanton, J. Am. Chem. Soc. 122 (2000) 939-949.

[28] R. J. Halter, R. L. Fimmen, R. J. McMahon, S. A. Peebles, R. L. Kuczkowksi, J. F. Stanton, J. Am. Chem. Soc. 123 (2001) 12353-12363.

[29] N. C. Craig, P. Groner, D. C. McKean, J. Phys. Chem. A 110 (2006) 7461-7469.

[30] D. McNaughton, D. N. Bruget, J. Mol. Struct. 273 (1992) 11-25.

[31] D. A. Matthews, J. Vázquez, J. F. Stanton, Mol. Phys. 105 (2007) 2659-2666.

[32] X. Zhang, M. R. Nimlos, G. B. Ellison, M. E. Varner, J. F. Stanton, J. Chem. Phys. 126 (2007) 174308

[33] I. M. Konen, E. X. J. Li, M. I. Lester, J. Vázquez, J. F. Stanton, J. Chem. Phys. 125 (2006) 074310

[34] J. M. L. Martin, P. R. Taylor, T. J. Lee, Chem. Phys. Lett. 275 (1997) 414-422

[35] J. M. L. Martin, T. J. Lee, P. R. Taylor, J.-P. Francois, J. Chem. Phys. 103 (1995) 2589-2602. 
Table 1

Experimental rotational constants $B_{0}$ and computed zero-point vibrational corrections $\Delta B_{0}$ (all values in MHz) for the various isotopologues of diacetylene.

\begin{tabular}{|c|c|c|c|c|c|c|}
\hline \multirow{3}{*}{$\begin{array}{l}\text { Isotopic } \\
\text { Species }\end{array}$} & \multirow[b]{3}{*}{$B_{0}$} & \multicolumn{5}{|c|}{$\Delta B_{0}$} \\
\hline & & (fc)MP2/ & $(\mathrm{fc}) \operatorname{CCSD}(\mathrm{T}) /$ & $(\mathrm{fc}) \operatorname{CCSD}(\mathrm{T}) /$ & $\operatorname{CCSD}(\mathrm{T}) /$ & $(\mathrm{fc}) \operatorname{CCSD}(\mathrm{T}) /$ \\
\hline & & cc-pVTZ & cc-pVTZ & cc-pVQZ & cc-pCVQZ & ANO2 \\
\hline $\mathrm{HCCCCH}$ & $4389.3019(39)^{d}$ & 2.098 & 0.912 & 1.922 & 1.838 & 2.083 \\
\hline DCCCCD & $3809.2433(66)^{e}$ & -0.207 & -1.110 & -0.203 & -0.237 & -0.005 \\
\hline $\mathrm{H}^{13} \mathrm{C}^{13} \mathrm{C}^{13} \mathrm{C}^{13} \mathrm{CH}$ & $4098.8959(36)^{e}$ & 2.145 & 1.064 & 1.970 & 1.890 & 2.108 \\
\hline $\mathrm{H}^{13} \mathrm{C}^{13} \mathrm{CCCH}$ & $4243.7325(111)^{e}$ & 2.130 & 0.997 & 1.953 & 1.871 & 2.103 \\
\hline $\mathrm{H}^{13} \mathrm{CCCCH}$ & $4258.5465(105)^{e}$ & 2.112 & 0.953 & 1.912 & 1.829 & 2.063 \\
\hline $\mathrm{HC}^{13} \mathrm{CCCH}$ & $4371.6291(45)^{e}$ & 2.113 & 0.955 & 1.962 & 1.879 & 2.121 \\
\hline $\mathrm{H}^{13} \mathrm{C}^{13} \mathrm{C}^{13} \mathrm{CCH}$ & $4224.7392(99)^{e}$ & 2.138 & 1.032 & 1.987 & 1.905 & 2.134 \\
\hline $\mathrm{H}^{13} \mathrm{C}^{13} \mathrm{CC}^{13} \mathrm{CH}$ & $4115.0556(42)^{e}$ & 2.133 & 1.027 & 1.935 & 1.855 & 2.075 \\
\hline HCCCCD & $4084.45342(7)^{f}$ & 0.815 & -0.219 & 0.745 & 0.688 & 0.927 \\
\hline $\mathrm{H}^{13} \mathrm{CCCCD}$ & $3964.11797(17)^{f}$ & 0.871 & -0.141 & 0.774 & 0.718 & 0.946 \\
\hline $\mathrm{HC}^{13} \mathrm{CCCD}$ & $4066.49893(16)^{f}$ & 0.830 & -0.178 & 0.782 & 0.725 & 0.962 \\
\hline $\mathrm{HCC}^{13} \mathrm{CCD}$ & $4071.64202(16)^{f}$ & 0.851 & -0.160 & 0.800 & 0.743 & 0.980 \\
\hline $\mathrm{HCCC}^{13} \mathrm{CD}$ & $3977.69016(15)^{f}$ & 0.870 & -0.144 & 0.775 & 0.718 & 0.947 \\
\hline
\end{tabular}

${ }^{a}$ Ref. (fc)CCSD(T)/cc-pVQZ

${ }^{c}$ Ref. (ae)CCSD(T)/cc-pCVQZ

${ }^{d}$ Ref. [20].

${ }^{e}$ Ref. [4].

${ }^{f}$ Ref. [5]. 
Table 2

Equilibrium structures of diacetylene and related molecules $(\AA)$.

\begin{tabular}{|c|c|c|c|}
\hline Method & $r_{\mathrm{C}-\mathrm{H}}$ & $r_{\mathrm{C} \equiv \mathrm{C}}$ & $r_{\mathrm{C}-\mathrm{C}}$ \\
\hline (fc)MP2/cc-pVTZ & 1.0620 & 1.2194 & 1.3687 \\
\hline (fc) $\operatorname{CCSD}(\mathrm{T}) / \mathrm{cc}-\mathrm{pVTZ}$ & 1.0638 & 1.2149 & 1.3789 \\
\hline (fc) $\operatorname{CCSD}(\mathrm{T}) / \mathrm{cc}-\mathrm{pVQZ}$ & 1.0633 & 1.2119 & 1.3769 \\
\hline (fc) $\operatorname{CCSD}(\mathrm{T}) / \mathrm{ANO} 2$ & 1.0631 & 1.2118 & 1.3766 \\
\hline$(\mathrm{fc}) \operatorname{CCSD}(\mathrm{T}) / \mathrm{cc}-\mathrm{pVQZ}+\operatorname{corr}(\text { Ref. }[6])^{a}$ & 1.0615 & 1.2087 & 1.3720 \\
\hline$(\mathrm{fc}) \operatorname{CCSD}(\mathrm{T}) / \mathrm{cc}-\mathrm{pV} 5 \mathrm{Z}$ & 1.0630 & 1.2111 & 1.3764 \\
\hline (fc) $\operatorname{CCSD}(T) / c c-p V 6 Z$ & 1.0630 & 1.2109 & 1.3762 \\
\hline $\operatorname{CCSD}(\mathrm{T}) / \mathrm{cc}-\mathrm{pCVTZ}$ & 1.0633 & 1.2121 & 1.3770 \\
\hline $\operatorname{CCSD}(\mathrm{T}) / \mathrm{cc}-\mathrm{pwCVTZ}$ & 1.0630 & 1.2111 & 1.3763 \\
\hline $\operatorname{CCSD}(\mathrm{T}) / \mathrm{cc}-\mathrm{pCVQZ}$ & 1.0621 & 1.2091 & 1.3742 \\
\hline $\operatorname{CCSD}(\mathrm{T}) / \mathrm{cc}-\mathrm{pwCVQZ}$ & 1.0620 & 1.2089 & 1.3741 \\
\hline (fc) $\operatorname{CCSD}(\mathrm{T}) / \mathrm{cc}-\mathrm{pwCVQZ}$ & 1.0632 & 1.2115 & 1.3766 \\
\hline $\operatorname{CCSD}(\mathrm{T}) / \mathrm{cc}-\mathrm{pCV} 5 \mathrm{Z}$ & 1.0617 & 1.2083 & 1.3737 \\
\hline$r_{0}$ & 1.0561 & 1.2079 & 1.3752 \\
\hline$\left(r_{m}^{\rho}\right)_{c o r r}($ Ref. [4]) & $1.0613(1)$ & $1.2096(1)$ & $1.3708(2)$ \\
\hline$r_{e}(\operatorname{Ref} .[5])$ & 1.0609 & 1.2104 & 1.3709 \\
\hline$r_{e}^{e m p}((\mathrm{fc}) \mathrm{MP} 2 / \mathrm{cc}-\mathrm{pVTZ})$ & 1.0623 & 1.2077 & 1.3736 \\
\hline$r_{e}^{e m p}((\mathrm{fc}) \operatorname{CCSD}(\mathrm{T}) / \mathrm{cc}-\mathrm{pVTZ})$ & 1.0620 & 1.2083 & 1.3732 \\
\hline$r_{e}^{e m p}((\mathrm{fc}) \operatorname{CCSD}(\mathrm{T}) / \mathrm{cc}-\mathrm{pVQZ})$ & 1.0616 & 1.2084 & 1.3727 \\
\hline$r_{e}^{e m p}(\operatorname{CCSD}(\mathrm{T}) / \mathrm{cc}-\mathrm{pCVQZ})$ & 1.0615 & 1.2085 & 1.3727 \\
\hline$\underline{r e}_{e}^{e m p}((\mathrm{fc}) \operatorname{CCSD}(\mathrm{T}) / \mathrm{ANO} 2)$ & 1.0614 & 1.2084 & 1.3726 \\
\hline$r_{e}^{e m p}$ Acetylene (Ref. [24]) & 1.0618 & 1.2029 & - \\
\hline$r_{e}^{e m p} \mathrm{HC}_{3} \mathrm{~N}$ (Ref. [25]) & 1.0623 & 1.2059 & 1.3761 \\
\hline$r_{e}^{e m p} \mathrm{HC}_{4} \mathrm{~F}(\text { Ref. }[6])^{b}$ & 1.0614 & 1.2080 & 1.3731 \\
\hline$r_{e}^{e m p} \mathrm{H}_{3} \mathrm{C}-\mathrm{C}_{4}-\mathrm{H}$ (Ref. [23]) & $1.6013(3)$ & $1.2085(6) / 1.2091(16)$ & $1.3734(14)$ \\
\hline
\end{tabular}


Table 3

Vibrational fundamentals of diacetylene $\left(\mathrm{in}^{-1}\right)$.

\begin{tabular}{|c|c|c|c|c|c|c|c|c|c|c|c|}
\hline & Experiment $^{b}$ & (fc)MP & /cc-pVTZ & $(\mathrm{fc}) \mathrm{CC}$ & Г)/cc-pVTZ & $(\mathrm{fc}) \mathrm{CC}$ & T)/cc-pVQZ & CCSD & /cc-pCVQZ & $(\mathrm{fc}) \mathrm{CC}$ & $(\mathrm{T}) / \mathrm{ANO} 2$ \\
\hline & & harm. & anharm. & harm. & anharm. & harm. & anharm. & harm. & anharm. & harm. & anharm. \\
\hline$\nu_{1}\left(\Sigma_{g}^{+}\right)$ & 3332 & 3486 & 3361 & 3458 & 3329 & 3457 & 3281 & 3463 & 3333 & 3462 & 3330 \\
\hline$\nu_{3}\left(\Sigma_{g}^{+}\right)$ & 872 & 897 & 867 & 887 & 849 & 892 & 855 & 894 & 865 & 891 & 861 \\
\hline$\nu_{4}\left(\Sigma_{u}^{+}\right)$ & 3334 & 3482 & 3369 & 3454 & 3339 & 3458 & 3288 & 3465 & 3338 & 3461 & 3329 \\
\hline$\nu_{6}\left(\Pi_{g}\right)$ & 626 & 620 & 625 & 623 & 643 & 632 & 616 & 636 & 635 & 639 & 627 \\
\hline$\nu_{7}\left(\Pi_{g}\right)$ & 483 & 487 & 452 & 474 & 419 & 481 & 484 & 484 & 490 & 482 & 479 \\
\hline$\nu_{8}\left(\Pi_{u}\right)$ & 628 & 629 & 602 & 633 & 614 & 634 & 616 & 640 & 635 & 640 & 627 \\
\hline$\nu_{9}\left(\Pi_{u}\right)$ & 220 & 230 & 217 & 227 & 216 & 220 & 225 & 221 & 223 & 220 & 219 \\
\hline
\end{tabular}

The accuracy of the reported fundamental frequencies obtained using Dunning's quadruple-zeta basis sets is probably only a couple of wavenumbers, as it turned out impossible to converge the required CC second-derivative calculations at the displaced points with very tight convergence thresholds. ${ }^{b}$ Ref. [30] and references therein. 